\title{
Instrument Analysis of Elementary Students' Curiosity on Natural Science Subjects Using the Rasch Model
}

\author{
Nadia Aulia Nadhirah ${ }^{1, *}$, Dindin Abdul Muiz Lidzinillah ${ }^{2}$, Nurul Hadi Yanti ${ }^{2}$, Aslina Binti Ahmad ${ }^{3}$ \\ ${ }^{1}$ Department of Guidance and Counseling, Universitas Pendidikan Indonesia, Indonesia \\ ${ }^{2}$ Department of Elementary School Teachers, Universitas Pendidikan Indonesia, Indonesia \\ ${ }^{3}$ Department of Psychology and Counseling, Universiti Pendidikan Sultan Idris, Malaysia
}

Received May 11, 2020; Revised July 12, 2020; Accepted July 20, 2020

\section{Cite This Paper in the following Citation Styles}

(a): [1] Nadia Aulia Nadhirah, Dindin Abdul Muiz Lidzinillah, Nurul Hadi Yanti, Aslina Binti Ahmad, "Instrument Analysis of Elementary Students' Curiosity on Natural Science Subjects Using the Rasch Model," Universal Journal of Educational Research, Vol. 8, No. 10, pp. 4897 - 4908, 2020. DOI: 10.13189/ujer.2020.081062.

(b): Nadia Aulia Nadhirah, Dindin Abdul Muiz Lidzinillah, Nurul Hadi Yanti, Aslina Binti Ahmad (2020). Instrument Analysis of Elementary Students' Curiosity on Natural Science Subjects Using the Rasch Model. Universal Journal of Educational Research, 8(10), 4897- 4908. DOI: 10.13189/ujer.2020.081062.

Copyright $\bigcirc 2020$ by authors, all rights reserved. Authors agree that this article remains permanently open access under the terms of the Creative Commons Attribution License 4.0 International License

\begin{abstract}
This research is motivated by the lack of curiosity of elementary school students in studying natural science subjects that affect the low quality of student learning. Until now there has been no research that examines the role of dimensions/indicators of curiosity. Thus, research on this matter must be carried out. This research is supported by using the Rasch model as a medium for testing. The purpose of this study is to show a description of the evaluation of curiosity of elementary school students in grades 5 and 6 of science subjects with the Rasch model to measure the accuracy of the instrument. The research participants were 99 participants from schools in Tasikmalaya. Data collection tools in the form of a self-report questionnaire to measure student curiosity. The results of the study were analyzed using the Rasch model and acceptable reliability (Alpha Cronbach $=0.74$ ) was included in either category. Furthermore, the value of Person Reliability is 0.70 as an indicator of the consistency of respondents' answers, including sufficient categories. Because as many as 94 elementary school students in grades 5 and 6 were declared fit, in the sense of giving answers according to ability level. While 5 other students answered less according to their ability level, in the sense of the possibility of guessing an answer or not giving an answer. While the Item Reliability of 0.88 as an indicator of the quality of items in the instrument, is classified as a good category.
\end{abstract}

Keywords Elementary School, Curiosity, Natural Sciences, Testing, Rasch Models, Quality of Learning

\section{Introduction}

Education has an important role in improving the quality of human resources. Education today is a future investment. One aim is to shape the character of students. Character education is very important to do [1], [2]. As a major investment, education needs to hone students' curiosity. Increasing everyone's curiosity is very important, it is a fuel/intrinsic motivation that has great potential to increase intelligence in student learning [3]-[9]. Also, curiosity makes students an active observer, gets rid of boredom, challenges students to keep on searching for new things, and as an adaptive process related to the pursuit of novelty or challenge [7], [10], [11]. According to Loewenstein, there are two types of curiosity, state curiosity, and trait curiosity. State curiosity refers to the curiosity that arises in certain situations, while trait curiosity refers to the capacity or tendency of a person to experience curiosity itself. The curiosity of students must always be fostered by teachers so that their vocabulary of knowledge continues to develop [12]-[14]. Curiosity becomes stronger when the information gap becomes smaller [15]. Meanwhile, Daniel Berlyne put curiosity on two axes by making four dimensions. The desire for information and knowledge and perception that describes a person's attention to new objects in the immediate environment. On the other axis, there is Specific Absorption, which is the desire for a 
specific observation, and Diverse-Exploration, which is the curiosity that drives a person to look for a stimulus to get out of boredom [13], [14], [16].

Elementary school students are required to master the competencies of each subject in the basic education curriculum. Science is one of the subjects in the curriculum [17] Almost all subjects in the school need the role of curiosity in the learning process of students, one of the subjects that emphasize that role is the science subject that applies scientific learning, in which there is a practicum process that focuses on student learning efforts to train children to make scientific decisions and take responsibility for those decisions accompanied by the impetus of students exploration, experimentation and application related to curiosity, self-study and the environment, as well as further development in the application of daily life [17], [18]. Individuals who have high curiosity tend to be easier to recognize, pursue, and become preoccupied with new and challenging experiences [9]. Curiosity research is limited to measuring children's curiosity behavior in response to stimuli that arouse curiosity (e.g. toys, games) when practicum in the laboratory, not in daily classroom practice. Also, the proposed definition of curiosity often confuses the concept of curiosity with the concepts of interest, intelligence, or motivation, which makes it unclear what is measured or what mechanism might underlie children's curiosity behavior [8].

Based on several studies it can also be seen that the lack of curiosity in science subjects will have an impact on problem-solving skills and student learning achievement [19]. The attitude is shown by students with a lack of curiosity when the learning process is only to hear the explanation from the teacher tends to be quiet and less active in asking questions, many students are silent and not responding to their teacher, playing alone, not focusing on reading the material in books and learning processes [17], [20]. Thus, Kashdan identified several characteristics of students with a lack of curiosity towards learning to include; 1) students have difficulty in the order showed in failure to understand the concept of time, and 2) students will lose track of their assignments and attendance to the concentrate, and students have experience of cognitive confusion in the presence of disruptive stimuli, show poor organizational skills, and find it difficult to return focus after losing concentration [15], [21].

Until now there has been no research that examines the role of dimensions/indicators of curiosity [22]. Thus, research on science education must be expanded, and the effectiveness of various methods of science education and its contribution to the development of scientific thought must be compared [23]. Based on the problem and the results of several studies, this study seeks to identify more about the curiosity of elementary school students in grades 5 and 6 in science subjects with Rasch's modeling approach through the Winstep program. The advantages of Rasch modeling compared to other methods is the ability to predict missing data and the interaction between respondent-items in the instrument using the same logit scale interval and being able to analyze polytomy items and be able to show responses to the statistical independence of some items [24]-[26]. Rasch is an excellent model for analyzing the validity of an instrument. But, there are important things to consider. The number of study participants and the number of parameters measured for each item. Too many parameters with few respondents will cause errors in the data [27].

This research is one of the initial explorations that aims to determine the accuracy and success of the curiosity instruments of elementary school students through the Rasch model as an assessment of learning outcomes. The results of this study are expected to be one of the results of the evaluation of input (input) about the personality of elementary school students and benchmarks for the management of study programs to assess the success of the learning process.

The impact of this study can determine the level of children's curiosity in science subjects so that it becomes material for teachers in the evaluation of further learning.

This research will answer the following questions:

1. How is the distribution of instruments that express students' curiosity in science subjects using the Rasch model?

2. Why can the Rasch Model express students' curiosity in science subjects in elementary school?

\section{Research Methodology}

The study used in a quantitative approach, with descriptive methods about actual problems through the process of collecting, compiling, or classifying, processing, and interpreting data by examining the relationship between variables and research instruments [29] through curiosity assessment instruments in the form of valence and factual statements. A valence statement is a statement to uncover students' attitudes towards the picture of the learning situation while a factual statement is to find out the behavior of students towards the problem that occurs [24]. The study population was elementary school students grade 5 and 6 at SDN Dadaha and MIN 1 Tasikmalaya. We take grades 5 and 6 because they have quite mature characteristics, they also have enough level of concentration in learning that increases and tends to increase. They have begun to pay attention to the time in doing something, start reading factual things and start thinking all with the character of the instruments we make [30], [31]. The opportunity to answer the instrument statement depends on the ratio between one's ability and the difficulty level of the problem. The following population details are presented in table 1 . 
Table 1. Research Samples

\begin{tabular}{|c|c|c|c|c|}
\hline \multirow{2}{*}{ School Name } & \multirow{2}{*}{ Class } & \multicolumn{2}{|c|}{ Population } & \multirow{2}{*}{ Total } \\
\cline { 3 - 4 } & & $\mathbf{L}$ & $\mathbf{P}$ & \\
\hline SDN Dadaha & $5 \mathrm{~A}$ & 14 & 12 & 26 \\
\hline SDN Dadaha & $5 \mathrm{~B}$ & 11 & 11 & 22 \\
\hline MIN 1 Tasikmalaya & $5 \mathrm{~A}$ & 13 & 8 & 21 \\
\hline MIN 1 Tasikmalaya & $5 \mathrm{~B}$ & 12 & 9 & 21 \\
\hline MIN 1 Tasikmalaya & $6 \mathrm{~A}$ & 3 & 6 & 9 \\
\hline Total & & $\mathbf{5 3}$ & $\mathbf{4 6}$ & $\mathbf{9 9}$ \\
\hline
\end{tabular}

The instrument in the form of a questionnaire was used in this study to express students' curiosity. There are a total of 13 items, which are adjusted with indicators/dimensions of curiosity including epistemic curiosity, perception curiosity, specific curiosity, and diverse curiosity. The types of questions are presented in the form of statements and are answered on a certain scale according to the level of curiosity students have.

The steps in this research are: a) making a statement of the problem, b) looking for a theoretical foundation, c) formulating a hypothesis, d) collecting data, e) conducting data analysis, and f) concluding.

\section{Results}

Instrument Assessment regarding the measurement of elementary school students' curiosity using the Rasch Model Application with Winstep Program 4.4.5.

The results of data processing instruments are as follows:

\subsection{Development of Analysis Instrumen}

From Table 2, the results of data analysis show that Raw variance explained by measures of $29 \%$ is included in enough category. Whereas the Unexplained variance in the 1 st to the 5 th contrast of residuals was $16.4 \%, 12.7 \%$, $11.2 \%, 9.3 \%$, and $8.9 \%$. It appears that the value of unexplained variance in the 1 st is more than $15 \%$. This indicates that this instrument has not measured the variables to be measured, to measure the level of student curiosity in elementary schools.

Table 2. Unidimensionality

\begin{tabular}{|c|c|c|c|}
\hline $\begin{array}{c}\text { Raw } \\
\text { Unexplained } \\
\text { Variance }\end{array}$ & Eigenvalue & Observed & Expected \\
\hline $\begin{array}{c}\text { Unexplained } \\
\text { variance in 1st }\end{array}$ & 2.1281 & $11.6 \%$ & $16.4 \%$ \\
\hline $\begin{array}{c}\text { Unexplained } \\
\text { variance in 2nd }\end{array}$ & 1,6482 & $9.0 \%$ & $12.7 \%$ \\
\hline $\begin{array}{c}\text { Unexplained } \\
\text { variance in 3rd }\end{array}$ & 1.4570 & $8.0 \%$ & $11.2 \%$ \\
\hline $\begin{array}{c}\text { Unexplained } \\
\text { variance in 4th }\end{array}$ & 1.2098 & $6.6 \%$ & $9.3 \%$ \\
\hline $\begin{array}{c}\text { Unexplained } \\
\text { variance in 5th }\end{array}$ & 1.1613 & $6.3 \%$ & $8.9 \%$ \\
\hline
\end{tabular}

Table 3. Wright's Map (Person-item Map)

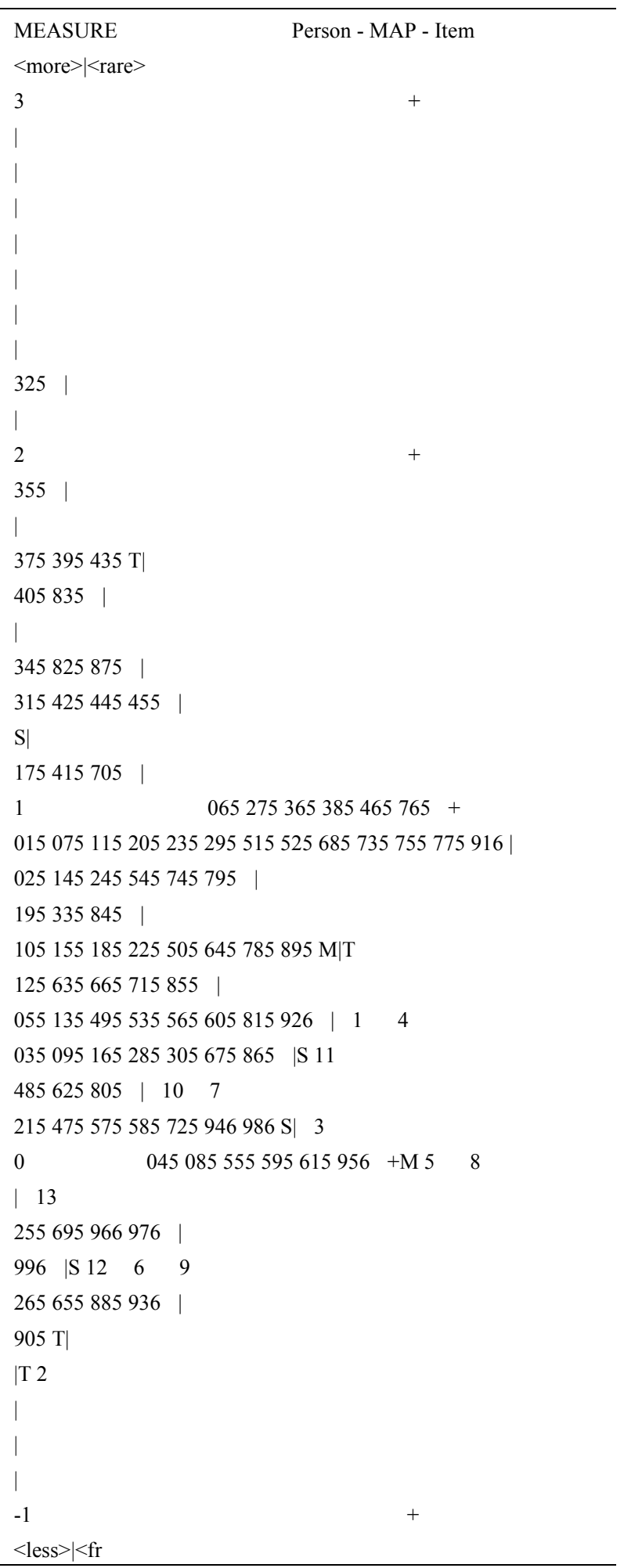

Table 3 can be seen that the map to determine the level of curiosity ability of students in elementary school spreads in the range of -1 to 3 logits. Their ability positions are mostly between $-2 \mathrm{SD}$ and $+2 \mathrm{SD}$. The average logit of elementary school students' curiosity ability of +0.62 (check the output of the Table 17 Measure Order in the appendix) is above the average logit item of 0.00 . This 
means that the average logit curiosity ability of elementary school students is above the average level of difficulty of standard items.

Meanwhile, the item difficulty level map is spread in the range -1 to 1 logit. Difficulty level position of 13 items (questions) is between $-2 \mathrm{SD}-+2 \mathrm{SD}$. The average level of difficulty of standard the items is below the level of students' curiosity abilities. Thus, the instrument items know the level of curiosity ability of students in elementary school is easily approved by elementary school students.

\subsection{Item Analysis}

To find out the level of difficulty items can be examined from Table 4: Item Measure. From this table, it is known that the $\mathrm{SD}$ value is 0.30 . With a limit on values for the very difficult category is more than 0.30 , the hard category is 0.00 to 0.30 , the easy category is -0.30 to less than 0.00 , and the very easy category is less than -0.30 .

Table 4. Item Measure

\begin{tabular}{|c|c|c|}
\hline Entry Number & Measure & Item \\
\hline 4 & 0.42 & Q4 \\
\hline 1 & 0.37 & Q1 \\
\hline 11 & 0.33 & Q11 \\
\hline 10 & 0.24 & Q10 \\
\hline 7 & 0.16 & Q7 \\
\hline 3 & 0.09 & Q3 \\
\hline 5 & 0.02 & Q5 \\
\hline 8 & -0.01 & Q13 \\
\hline 13 & -0.11 & Q6 \\
\hline 6 & -0.28 & Q9 \\
\hline 9 & -0.29 & Q12 \\
\hline 12 & -0.34 & Q2 \\
\hline 2 & -0.58 & \\
\hline MEAN & 0.00 & \\
\hline SD & 0.30 & \\
\hline & & \\
\hline & & \\
\hline
\end{tabular}

Table 5. Items Fit

\begin{tabular}{|c|c|c|c|c|c|}
\hline \multirow{2}{*}{$\begin{array}{c}\text { Entry } \\
\text { Number }\end{array}$} & \multirow{2}{*}{ Measure } & \multicolumn{2}{|c|}{ OUTFIT } & \multirow{2}{*}{$\begin{array}{c}\text { PT } \\
\text { Measure } \\
\text { Corr } \\
\end{array}$} & \multirow{2}{*}{ Item } \\
\hline & & MNSQ & ZSTD & & \\
\hline 2 & -0.58 & 1.39 & 2.01 & 0.36 & Q5 \\
\hline 5 & 0.02 & 1.39 & 2.56 & 0.48 & Q5 \\
\hline 7 & 0.16 & 1.21 & 1.52 & 0.44 & Q7 \\
\hline 11 & 0.33 & 1.06 & 0.50 & 0.36 & Q11 \\
\hline 13 & -0.11 & 0.95 & -0.32 & 0.45 & Q13 \\
\hline 6 & -0.28 & 0.93 & -0.41 & 0.59 & Q6 \\
\hline 3 & 0.09 & 0.95 & -0.36 & 0.32 & Q3 \\
\hline 8 & -0.01 & 0.94 & -0.38 & 0.50 & Q8 \\
\hline 10 & 0.24 & 0.92 & -0.61 & 0.51 & Q10 \\
\hline 4 & 0.42 & 0.90 & -0.81 & 0.58 & Q4 \\
\hline 12 & -0.34 & 0.89 & -0.68 & 0.54 & Q12 \\
\hline 9 & -0.29 & 0.81 & -1.22 & 0.54 & Q9 \\
\hline 1 & 0.37 & 0.71 & -2.47 & 0.64 & Q1 \\
\hline
\end{tabular}

By looking at the logit value of each item in the Table 5 column Measure known that 3 items are included in the very difficult category, namely items number 4,1 , and 11 The hard category contains 4 items, namely numbers $10,7,3$ and 5. Easy categories, there are 4 items, namely $8,13,6$ and 9 . While the category is very easy there are 2 items, namely numbers 12 and 2 .

An item can be considered fit if it meets at least 1 of the 3 criteria.

Based on the 1st criteria, there are no misfit items. According to the 2 nd standard, there are 3 misfit items, namely numbers 5,2 , and 1 . Whereas based on the $3 \mathrm{rd}$ criteria, it is known that as many as 10 items having a PT Measure Corr value of more than 0.4 and less than 0.85 there are 3 items, namely numbered 2, 11 and 3 which have the value of PT Measure Corr in succession as follows: $0,36,0,36$ and 0,32 or less than 0,4 , so they are stated as the outfit. Referring to the views of Booner et al. (2014) than 13 items of statement items to find out the students' curiosity in elementary schools are declared fit in the sense of functioning normally and can be understood correctly by students in elementary schools and can measure what must be measured in this case is student curiosity.

Table 6. Rating Scale Diagnostics

\begin{tabular}{|c|c|c|}
\hline $\begin{array}{c}\text { Category } \\
\text { Label }\end{array}$ & Observed Average & Andrich Threshold \\
\hline 1 & -0.01 & None \\
\hline 2 & 0.18 & -0.62 \\
\hline 3 & 0.37 & -0.47 \\
\hline 4 & 0.71 & 0.64 \\
\hline 5 & 1.01 & 0.46 \\
\hline
\end{tabular}

In Table 6. it is known that the observed average value and Andrich Threshold show conformity and are equally increased in alternative answers 1,2, 3, and 5 while alternative answer 4 confuses respondents because of the inconsistency of Andrich Threshold's value. Thus, it can be stated that the differences in the choice of answers 1, 2, 3, and 5 can be understood by participants, while the choice of answers 4 can not be understood. Thus, alternative instrument answers are suggested not five but enough four.

Table 7. Detection of Bias Items

\begin{tabular}{|c|c|}
\hline Probability & Item Number \\
\hline 1.0000 & Q1 \\
\hline 0,0007 & Q2 \\
\hline 0.2683 & Q3 \\
\hline 0.3619 & Q4 \\
\hline 0.0518 & Q5 \\
\hline 0.0095 & Q6 \\
\hline 0.5494 & Q7 \\
\hline 0.7470 & Q8 \\
\hline 0.1288 & Q9 \\
\hline 0.3443 & Q10 \\
\hline 0.0868 & Q11 \\
\hline 0.2237 & Q12 \\
\hline 1.0000 & Q13 \\
\hline
\end{tabular}


The results of the analysis of bias based on gender can be seen that 2 statement numbers have a gender bias, namely numbers 2 and 6 with a probability value of less than 0.05 namely 0.0007 and 0.0095 . An overall picture of the logit position for each item by gender can be seen in the following figure.

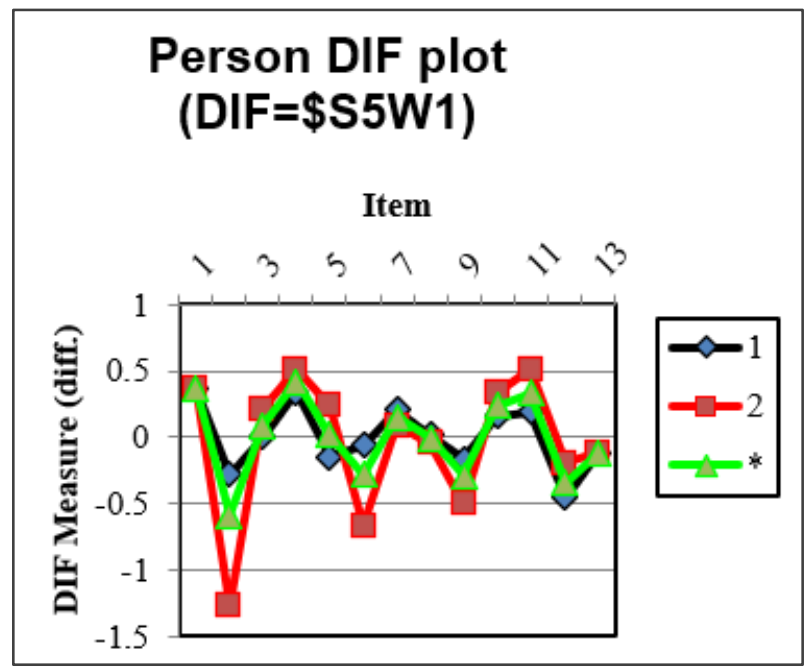

Figure 1. DIF person

From the picture, it appears that item number 2 and 6 are easier for female students to work on so that they benefit female students and tend to disadvantage male students.

\subsection{Analysis of Elementary School Student Ability}

The results of the study describe empirical data about the Curiosity of Elementary School students grade 5 and 6 at SDN Dadaha and MIN 1 Tasikmalaya, which can be seen in table 8 .

Table 8. Student Ability

\begin{tabular}{|c|c|c|}
\hline Category & Frequency & Proportion \\
\hline High & 14 & 14,14 \\
\hline Middle & 69 & 69.69 \\
\hline Low & 16 & 16,16 \\
\hline Total & 99 & 99.99 \\
\hline
\end{tabular}

Data on the individual ability of elementary school students in grades 5 and 6 can be found in Table 8. Person Measure. From this table, SD values are 0.56. This SD value when combined with an average logit (mean) value of 0.62 means that the individual abilities of Elementary School students in grades 5 and 6 can be grouped into high ability categories (greater than $0.62+0.56=1.18$ ), moderate ability categories (between $0.62-0.56$ and $0.62+$ 0.56 or 0.06 and 1.18), and low ability categories (less than $0.62-0.56=0.06$ ). Thus, the logit value limit for the high ability category is more than 1.18 , the medium ability category starts from 0.06 to 1.18 , and the low ability category is less than 0.06 .

In table 8 shows that the level of curiosity of students in grades 5 and 6 at SDN Dadaha and [MIN 1 in Tasikmalaya] in science subjects is mostly at a moderate level $(69.69 \%)$, meaning that students in grades 5 and 6 at SDN Dadaha and MIN 1 Tasikmalaya tends to hesitate to understand the views of others in an instrument condition. By looking at the logit value of each grade 5 and 6 elementary school students in Table 3 Measure column, respectively based on the level of ability of the participants as many as 99 people, it is known that there are 14 people included in the category of high ability, the category of medium ability there are 69 people and 16 people categories low ability.

\subsection{Person Fit}

To see the suitability of an individual's response based on his or her ability with the ideal model (person fit) can be examined based on the data in the output Criteria for checking the suitability of the person (person fit) or person incompatibility (outlier or misfit) according to Booner et al. (2014) are as follows: (1) $0.5<\mathrm{MNSQ}<1.5$ (the closer to 1 the better); (2) $-2.0<$ ZSTD $<+2.0$ (the closer to 0 the better); and (3) $0.4<$ PT MEASURE CORR $<0.85$. A participant can be considered fit if it meets at least 1 of the 3 criteria. Based on these criteria from the output of figure 2 (person fit) it can be seen that as many as 94 elementary school students in grades 5 and 6 are declared fit in the sense of giving answers according to their level of ability. While 5 other students gave an answer that was not according to their level of ability not answering, i.e. numbers: $12,27,65,89$, and 48. 
Person STATISTICS: MISFIT ORDER

\begin{tabular}{|c|c|c|c|c|c|c|c|c|c|c|c|c|c|}
\hline \multirow{2}{*}{$\begin{array}{l}\text { | ENTRY } \\
\text { | NUMBER }\end{array}$} & \multirow{2}{*}{$\begin{array}{l}\text { TOTAL } \\
\text { SCORE }\end{array}$} & \multirow{2}{*}{$\begin{array}{l}\text { TOTAL } \\
\text { COUNT }\end{array}$} & & MODEL | & & LNFIT & ou & TFIT & | PTMEAS & UR-AL | & EXACT & МАTCH| & \\
\hline & & & MEASURE & S.E. & |MNSQ & ZSTDI & |MNSQ & ZSTD| & |CORR. & EXP. I & OBS\% & EXP\%| & Person \\
\hline 16 & 43 & 13 & .26 & $.24 \mid$ & 2.74 & $3.84 \mid$ & | 2.74 & $3.82 \mid$ & |A. .06 & $.32 \mid$ & .0 & $32.4 \mid$ & 16511 \\
\hline 77 & 52 & 13 & .86 & .28 & 1.95 & $2.14 \mid$ & 2.62 & 3.09 & | B-. 51 & $.27 \mid$ & 7.7 & 26.9 & 77521 \\
\hline 12 & 47 & 13 & .51 & .25 & 1.92 & $2.31 \mid$ & 2.15 & 2.69 & |C.13 & $.31 \mid$ & 30.8 & 30.01 & 12511 \\
\hline 27 & 54 & 13 & 1.02 & .29 & 2.05 & $2.17 \mid$ & 2.09 & 2.15 & |D-.07 & $.26 \mid$ & 23.1 & 30.21 & 27512 \\
\hline 65 & 32 & 13 & -.40 & $.25 \mid$ & 1.93 & $2.27 \mid$ & 2.05 & $2.47 \mid$ & $\mid E-.22$ & $.31 \mid$ & 15.4 & 32.31 & 65521 \\
\hline 41 & 55 & 13 & 1.11 & $.31 \mid$ & 1.88 & $1.83 \mid$ & 2.00 & $1.92 \mid$ & |F. .13 & $.25 \mid$ & 15.4 & 33.21 & 41511 \\
\hline 82 & 58 & 13 & 1.44 & $.36 \mid$ & 1.96 & $1.68 \mid$ & 1.58 & $1.12 \mid$ & |G .42 & $.21 \mid$ & 46.2 & 43.81 & 82522 \\
\hline 48 & 42 & 13 & .20 & .24 & 1.93 & $2.39 \mid$ & 1.89 & $2.31 \mid$ & |H. .35 & .321 & 7.7 & $32.4 \mid$ & 48512 \\
\hline 89 & 49 & 13 & .64 & $.26 \mid$ & 1.93 & $2.26 \mid$ & 1.93 & $2.20 \mid$ & II .14 & $.29 \mid$ & 15.4 & 28.81 & 89521 \\
\hline 60 & 46 & 13 & .45 & $.25 \mid$ & 1.84 & 2.18 & 1.89 & $2.24 \mid$ & |了 . .44 & $.31 \mid$ & 15.4 & 30.61 & 60521 \\
\hline 5 & 45 & 13 & .38 & .25 & 1.84 & $2.19 \mid$ & 1.79 & $2.06 \mid$ & IK .46 & $.31 \mid$ & 23.1 & $31.8 \mid$ & 05511 \\
\hline 44 & 57 & 13 & 1.32 & $.34 \mid$ & 1.68 & $1.37 \mid$ & 1.50 & $1.04 \mid$ & IL .12 & .221 & 30.8 & 40.21 & 44511 \\
\hline 13 & 45 & 13 & .38 & $.25 \mid$ & 1.64 & $1.76 \mid$ & 1.62 & $1.71 \mid$ & M. .32 & $.31 \mid$ & 23.1 & $31.8 \mid$ & 13511 \\
\hline 34 & 58 & 13 & 1.44 & $.36 \mid$ & 1.61 & $1.21 \mid$ & |1.21 & $.55 \mid$ & N . .56 & $.21 \mid$ & 61.5 & 43.81 & 34511 \\
\hline 25 & 35 & 13 & -.22 & $.25 \mid$ & 1.52 & $1.47 \mid$ & 1.56 & $1.56 \mid$ & $10-.02$ & .321 & 23.1 & 32.81 & 25511 \\
\hline 78 & 48 & 13 & .57 & $.26 \mid$ & 1.49 & $1.37 \mid$ & 1.56 & $1.52 \mid$ & |P .17 & $.30 \mid$ & 7.7 & $29.4 \mid$ & 78521 \\
\hline 33 & 50 & 13 & .71 & $.27 \mid$ & 1.43 & $1.19 \mid$ & 1.50 & $1.32 \mid$ & Q-.35 & $.29 \mid$ & 23.1 & $28.6 \mid$ & 33511 \\
\hline 67 & 44 & 13 & .32 & .25 & 11.47 & $1.38 \mid$ & 1.49 & $1.42 \mid$ & |R .06 & $.32 \mid$ & 7.7 & $31.9 \mid$ & 67521 \\
\hline 19 & 50 & 13 & .71 & $.27 \mid$ & 1.42 & 1.19 & 1.36 & $1.02 \mid$ & S. .28 & .29 & 15.4 & 28.61 & 19512 \\
\hline 26 & 32 & 13 & -.40 & $.25 \mid$ & 1.36 & $1.06 \mid$ & | 1.42 & $1.18 \mid$ & |T .17 & $.31 \mid$ & 23.1 & 32.31 & 26511 \\
\hline 69 & 36 & 13 & -.16 & $.25 \mid$ & 1.39 & $1.17 \mid$ & 1.39 & $1.16 \mid$ & $U-.05$ & .321 & 38.5 & 32.61 & 69521 \\
\hline 7 & 52 & 13 & .86 & .28 & 1.24 & $.73 \mid$ & 1.38 & $1.02 \mid$ & V .14 & $.27 \mid$ & 23.1 & $26.9 \mid$ & 07512 \\
\hline 90 & 31 & 13 & -.47 & $.26 \mid$ & 1.35 & $1.03 \mid$ & |1.37 & $1.07 \mid$ & $w-.14$ & $.31 \mid$ & 30.8 & $32.1 \mid$ & 90522 \\
\hline 22 & 49 & 13 & .64 & $.26 \mid$ & 1.28 & $.86 \mid$ & 1.33 & $.96 \mid$ & $X-.10$ & $.29 \mid$ & 30.8 & 28.81 & 22512 \\
\hline 24 & 51 & 13 & .78 & $.27 \mid$ & 1.33 & $.96 \mid$ & 1.22 & $.68 \mid$ & Y .41 & $.28 \mid$ & 23.1 & $27.5 \mid$ & 24511 \\
\hline 1 & 52 & 13 & .86 & $.28 \mid$ & 1.29 & $.84 \mid$ & 1.23 & $.68 \mid$ & $Z .28$ & $.27 \mid$ & 23.1 & $26.9 \mid$ & 01511 \\
\hline 57 & 41 & 13 & .14 & $.24 \mid$ & 1.28 & $.91 \mid$ & 1.29 & $.94 \mid$ & .14 & $.32 \mid$ & 23.1 & 32.21 & 57521 \\
\hline 88 & 32 & 13 & -.40 & $.25 \mid$ & 1.27 & $.83 \mid$ & 1.24 & $.75 \mid$ & .57 & $.31 \mid$ & 23.1 & $32.3 \mid$ & 88522 \\
\hline 56 & 45 & 13 & .38 & .251 & 11.02 & .161 & 11.09 & .381 & -.19 & $.31 !$ & 30.8 & $31.8 !$ & 56521 \\
\hline & TTER F & ITTING & NOT SHOWN & & $-\cdots$ & ---++ & $-\cdots$ & ---+ & & & & & \\
\hline 64 & 48 & 13 & .57 & $.26 \mid$ & .79 & $-.54 \mid$ & .84 & $-.39 \mid$ & .40 & $.30 \mid$ & 38.5 & $29.4 \mid$ & 64521 \\
\hline 87 & 58 & 13 & 1.44 & $.36 \mid$ & .82 & $-.21 \mid$ & .84 & $-.14 \mid$ & -.27 & $.21 \mid$ & 30.8 & 43.81 & 87521 \\
\hline 29 & 53 & 13 & .94 & .29 & .67 & $-.86 \mid$ & .81 & $-.37 \mid$ & .22 & $.27 \mid$ & 30.8 & 30.21 & 29511 \\
\hline 23 & 53 & 13 & .94 & .29 & .79 & $-.46 \mid$ & .80 & -.42 & .58 & .27 & 30.8 & $30.2 \mid$ & 23511 \\
\hline 40 & 59 & 13 & 1.57 & .38 & .76 & $-.31 \mid$ & .80 & -.19 & .11 & .20 & 61.5 & $54.5 \mid$ & 40512 \\
\hline 61 & 39 & 13 & .02 & .24 & .77 & $-.65 \mid$ & .79 & -.61 & -.13 & $.32 \mid$ & 38.5 & 33.31 & 61521 \\
\hline 35 & 61 & 13 & 1.92 & $.47 \mid$ & .64 & $-.42 \mid$ & .77 & $-.13 \mid$ & -.06 & $.16 \mid$ & 69.2 & $76.1 \mid$ & 35512 \\
\hline 54 & 51 & 13 & .78 & .27 & .74 & $-.66 \mid$ & .76 & -.59 & .71 & .28 & 15.4 & 27.51 & 54522 \\
\hline 68 & 52 & 13 & .86 & .28 & .75 & $-.63 \mid$ & .75 & $-.57 \mid$ & .21 & $.27 \mid$ & 23.1 & $26.9 \mid$ & 68521 \\
\hline 3 & 44 & 13 & .32 & .25 & .73 & $-.82 \mid$ & .73 & $-.81 \mid$ & .31 & $.32 \mid$ & 53.8 & $31.9 \mid$ & 03511 \\
\hline 73 & 53 & 13 & .94 & .29 & .71 & -.72 & .68 & -.78 & .50 & .27 & 46.2 & $30.2 \mid$ & 73522 \\
\hline 10 & 48 & 13 & .57 & .26 & .70 & -.90 & .69 & -.90 & .43 & $.30 \mid$ & 30.8 & $29.4 \mid$ & 10512 \\
\hline 15 & 48 & 13 & .57 & $.26 \mid$ & .70 & $-.89 \mid$ & .67 & $-.98 \mid$ & -.03 & $.30 \mid$ & 38.5 & $29.4 \mid$ & 15511 \\
\hline 20 & 53 & 13 & .94 & $.29 \mid$ & .70 & $-.77 \mid$ & .64 & $-.90 \mid$ & .72 & $.27 \mid$ & 30.8 & 30.21 & 20512 \\
\hline 36 & 54 & 13 & 1.02 & .29 & .69 & $-.76 \mid$ & .66 & $-.80 \mid$ & .20 & $.26 \mid$ & 46.2 & 30.21 & 36512 \\
\hline 38 & 54 & 13 & 1.02 & .29 & .69 & $-.76 \mid$ & .66 & $-.80 \mid$ & .20 & .26 & 46.2 & $30.2 \mid$ & 38512 \\
\hline 50 & 49 & 13 & .64 & $.26 \mid$ & .63 & $-1.13 \mid$ & .68 & $-.90 \mid$ & .29 & $.29 \mid$ & 30.8 & $28.8 \mid$ & 50522 \\
\hline 30 & 43 & 13 & .26 & .24 & .65 & $-1.14 \mid$ & .65 & $-1.13 \mid$ & -.30 & $.32 \mid$ & 38.5 & $32.4 \mid$ & 30511 \\
\hline 83 & 59 & 13 & 1.57 & $.38 \mid$ & .65 & $-.57 \mid$ & .59 & $-.66 \mid z$ & $z .44$ & .20 & 61.5 & $54.5 \mid$ & 83522 \\
\hline 98 & 41 & 13 & .14 & .24 & .65 & $-1.15 \mid$ & .65 & $-1.14 \mid y$ & $y .29$ & $.32 \mid$ & 23.1 & $32.2 \mid$ & 98621 \\
\hline 99 & 34 & 13 & -.28 & $.25 \mid$ & .64 & $-1.11 \mid$ & .65 & $-1.10 \mid x$ & $x .63$ & $.32 \mid$ & 23.1 & $32.7 \mid$ & 99621 \\
\hline 17 & 55 & 13 & 1.11 & $.31 \mid$ & .64 & $-.85 \mid$ & .60 & $-.93 \mid w$ & $w .73$ & $.25 \mid$ & 30.8 & $33.2 \mid$ & 17512 \\
\hline 72 & 41 & 13 & .14 & .24 & .64 & $-1.19 \mid$ & .64 & $-1.18 \mid v$ & $v .31$ & $.32 \mid$ & 30.8 & $32.2 \mid$ & 72521 \\
\hline 75 & 53 & 13 & .94 & .29 & .64 & $-.95 \mid$ & .61 & $-1.00 \mid u$ & u . .63 & .27 & 46.2 & $30.2 \mid$ & 75522 \\
\hline 2 & 51 & 13 & .78 & $.27 \mid$ & .58 & -1.27 & .61 & $-1.11 \mid t$ & $t .35$ & $.28 \mid$ & 23.1 & $27.5 \mid$ & 02511 \\
\hline 81 & 46 & 13 & .45 & $.25 \mid$ & .58 & $-1.40 \mid$ & .60 & $-1.30 \mid \mathrm{s}$ & s . .22 & $.31 \mid$ & 30.8 & $30.6 \mid$ & 81521 \\
\hline 32 & 62 & 13 & 2.17 & $.54 \mid$ & .59 & $-.40 \mid$ & .50 & $-.52 \mid r$ & $r .46$ & .14 & 76.9 & $81.2 \mid$ & 32511 \\
\hline 52 & 53 & 13 & .94 & $.29 \mid$ & .52 & $-1.40 \mid$ & .59 & $-1.10 \mid q$ & $q .57$ & $.27 \mid$ & 38.5 & 30.21 & 52522 \\
\hline 86 & 43 & 13 & .26 & $.24 \mid$ & .59 & $-1.40 \mid$ & .59 & $-1.38 \mid p$ & p. .65 & $.32 \mid$ & 38.5 & $32.4 \mid$ & 86521 \\
\hline 92 & 45 & 13 & .38 & $.25 \mid$ & .56 & $-1.51 \mid$ & .57 & $-1.45 \mid 0$ & 0. .75 & $.31 \mid$ & 30.8 & $31.8 \mid$ & 92622 \\
\hline 9 & 44 & 13 & .32 & $.25 \mid$ & .54 & $-1.62 \mid$ & .54 & $-1.61 \mid n$ & .61 & $.32 \mid$ & 38.5 & $31.9 \mid$ & 09511 \\
\hline 28 & 44 & 13 & .32 & .25 & .51 & $-1.78 \mid$ & .54 & $-1.63 \mid \mathrm{m}$ & $\mathrm{n}-.24$ & $.32 \mid$ & 46.2 & $31.9 \mid$ & 28511 \\
\hline 6 & 54 & 13 & 1.02 & .29 & .52 & $-1.35 \mid$ & .51 & $-1.30 \mid 1$ & 1.43 & $.26 \mid$ & 30.8 & $30.2 \mid$ & 06512 \\
\hline 43 & 60 & 13 & 1.73 & .421 & .49 & $-.87 \mid$ & .51 & $-.76 \mid k$ & k. .29 & $.18 \mid$ & 69.2 & 63.01 & 43512 \\
\hline 51 & 53 & 13 & .94 & $.29 \mid$ & .50 & $-1.49 \mid$ & | . .51 & $-1.39 \mid$ & j. .66 & $.27 \mid$ & 38.5 & 30.21 & 51522 \\
\hline 74 & 51 & 13 & .78 & .27 & .46 & $-1.81 \mid$ & .51 & -1.49 & i. .60 & $.28 \mid$ & 38.5 & $27.5 \mid$ & 74522 \\
\hline 37 & 60 & 13 & 1.73 & .421 & .47 & $-.93 \mid$ & .47 & $-.88 \mid$ & h . .38 & $.18 \mid$ & 69.2 & $63.0 \mid$ & 37512 \\
\hline 39 & 60 & 13 & 1.73 & $.42 \mid$ & .47 & $-.93 \mid$ & | .47 & $-.88 \mid$ & g . . 38 & $.18 \mid$ & 69.2 & 63.00 & 39512 \\
\hline 46 & 54 & 13 & 1.02 & .29 & .44 & $-1.69 \mid$ & | .42 & $-1.69 \mid$ & $f-.15$ & $.26 \mid$ & 61.5 & 30.21 & 46512 \\
\hline 63 & 47 & 13 & .51 & .25 & .43 & $-2.10 \mid$ & .42 & -2.12 & e. .49 & $.31 \mid$ & 69.2 & 30.01 & 63522 \\
\hline 59 & 39 & 13 & .02 & .24 & .39 & $-2.38 \mid$ & | . .41 & $-2.29 \mid$ & d .18 & $.32 \mid$ & 61.5 & $33.3 \mid$ & 59521 \\
\hline 80 & 42 & 13 & .20 & .24 & .37 & $-2.51 \mid$ & .38 & -2.45 & c. .50 & .321 & 53.8 & $32.4 \mid$ & 80521 \\
\hline 21 & 41 & 13 & .14 & $.24 \mid$ & .33 & $-2.77 \mid$ & .33 & $-2.77 \mid$ & b . .69 & .321 & 53.8 & 32.21 & 21511 \\
\hline 55 & 39 & 13 & .02 & $.24 \mid$ & .21 & $-3.70 \mid$ & | .21 & $-3.70 \mid$ & a .65 & $.32 \mid$ & 69.2 & $33.3 \mid$ & 55521 \\
\hline & & 130 & $6 ?$ & & $a$ & - & $1 \cdots$ & a & -1 & $\cdots$ & 341 & 33 이 & \\
\hline MEAN & 47.7 & 13.0 & .62 & .28 & 1.00 & $-.1 \mid$ & 1.00 & $.0 \mid$ & & & 34.1 & $33.9 \mid$ & \\
\hline P.SD & 7.6 & .0 & .56 & .05 & .48 & $1.3 \mid$ & | . .50 & $1.3 \mid$ & & & 16.2 & 9.51 & \\
\hline
\end{tabular}

Figure 2. Person Fit 


\subsection{Indicator-Based Development}

Based on the results of the study describe empirical data about the curiosity of elementary school students based on the indicators that can be seen in table 9 .

Table 9. Development of Basic Shiva's Curiosity Based on Indicators

\begin{tabular}{|c|c|c|c|c|c|}
\hline Indicator & $\begin{array}{l}\text { Item } \\
\text { bullet }\end{array}$ & KET & $\mathbf{H}$ & $\mathbf{M}$ & $\mathbf{L}$ \\
\hline \multirow{6}{*}{$\begin{array}{l}\text { Epistemic } \\
\text { curiosity }\end{array}$} & \multirow{2}{*}{1} & $\mathrm{~F}$ & 37 & 42 & 20 \\
\hline & & $\%$ & 37.37 & 42.42 & 20,20 \\
\hline & \multirow{2}{*}{2} & $\mathrm{~F}$ & 77 & 9 & 13 \\
\hline & & $\%$ & 77.77 & 9.09 & 13.13 \\
\hline & \multirow{2}{*}{3} & $\mathrm{~F}$ & 69 & 17 & 13 \\
\hline & & $\%$ & 69.69 & 17.17 & 13.13 \\
\hline \multirow{8}{*}{$\begin{array}{l}\text { Perceptual } \\
\text { curiosity }\end{array}$} & \multirow{2}{*}{4} & $\mathrm{~F}$ & 41 & 33 & 25 \\
\hline & & $\%$ & 41.41 & 33.33 & 25.25 \\
\hline & \multirow{2}{*}{11} & $F$ & 58 & 18 & 23 \\
\hline & & $\%$ & 58.58 & 18.18 & 23.23 \\
\hline & \multirow{2}{*}{12} & $\mathrm{~F}$ & 63 & 23 & 13 \\
\hline & & $\%$ & 63.63 & 23.23 & 13.13 \\
\hline & \multirow{2}{*}{13} & $F$ & 54 & 23 & 22 \\
\hline & & $\%$ & 54.54 & 23.23 & 22.22 \\
\hline \multirow{6}{*}{$\begin{array}{l}\text { Specific } \\
\text { curiosity }\end{array}$} & \multirow{2}{*}{5} & $\mathrm{~F}$ & 56 & 23 & 20 \\
\hline & & $\%$ & 56.56 & 23.23 & 20,20 \\
\hline & \multirow{2}{*}{9} & $\mathrm{~F}$ & 66 & 26 & 7 \\
\hline & & $\%$ & 66.66 & 26.26 & 7.07 \\
\hline & \multirow{2}{*}{7} & $\mathrm{~F}$ & 49 & 28 & 22 \\
\hline & & $\%$ & 49.49 & 28.28 & 22.22 \\
\hline \multirow{6}{*}{$\begin{array}{l}\text { Diverse } \\
\text { curiosity }\end{array}$} & \multirow{2}{*}{6} & $\mathrm{~F}$ & 43 & 33 & 23 \\
\hline & & $\%$ & 43.43 & 33.33 & 23.23 \\
\hline & \multirow{2}{*}{8} & $\mathrm{~F}$ & 65 & 21 & 13 \\
\hline & & $\%$ & 65.65 & 21.21 & 13.13 \\
\hline & \multirow{2}{*}{10} & $\mathrm{~F}$ & 66 & 17 & 16 \\
\hline & & $\%$ & 66.66 & 17.17 & 16,16 \\
\hline
\end{tabular}

The table shows that students' curiosity can be seen from the percentage of each indicator spread on four of each indicator in the order of perceptual curiosity, epistemic curiosity, diverse curiosity, and specific curiosity.

\subsection{Instrument Analysis}

For instrument analysis the information presented in table 9.

Person measure 0.62 logit indicates the average score of all participants is greater than the average item (where the average item is 0.00 logit) shows that the ability of participants, in general, is greater than the difficulty of the item items of the instrument. Table 9 (Statistical Summary) is obtained Cronbach Alpha value, which represents the interaction between person and item items as a whole, is 0.74, including the good category. This shows a high-reliability coefficient, and this scale produces a consistent and reliable measurement score. Furthermore, the value of Person Reliability was 0.70 as an indicator of the consistency of respondents' answers, including enough category. While the Item Reliability of 0.88 as an indicator of the quality of items in the instrument, classified as a good category. This shows that all thirteen items were quality items and the majority of the respondents' groups responded in earnest.

Last of the output Table 10 notes the separation for the person is 1.51 and for an item is 2.68. The greater the separation value, the better the overall quality of the person and instrument. Separation values are calculated more precisely through the formula: $\mathrm{H}=\{(4 \mathrm{x}$ separation $)+1\} /$ 3 . So it is obtained separation for a person is 2.35 rounded to 2 . While the value of separation for item 3.90 is rounded to 4 . This means that the study participants have a variety of abilities that can be categorized into 2 groups. Meanwhile, the level of difficulty items spread in 4 groups ranging from the easiest to the most difficult groups.

Table 10. Statistical Summary

\begin{tabular}{|c|c|c|c|c|c|}
\hline & measure & Elementary school & Separation & Reliability & 0.70 \\
\hline Person & 0.62 & 0.56 & 1.51 & 0.88 \\
\hline Item & 0.00 & 0.30 & 2.68 & 0.74 \\
\hline
\end{tabular}

Table 10.1. Summary of Person

\begin{tabular}{|c|c|c|c|c|}
\hline \multicolumn{2}{|c|}{ INFIT } & \multicolumn{2}{c|}{ OUTFIT } \\
\hline MNSQ & ZSTD & MNSQ & -0.03 & Measure \\
\hline 1.00 & -0.6 & 1.00 & 0.13 & 0.62 \\
\hline 0.05 & 0.13 & 0.05 & 1.30 & 0.06 \\
\hline 0.48 & 1.31 & 0.50 & 1.31 & 3.82 \\
\hline 0.48 & 1.31 & 0.50 & -36 \\
\hline 2.74 & 3.84 & 2.74 & -3.70 & -0.57 \\
\hline
\end{tabular}


Table 10.2. Summary of Items

\begin{tabular}{|c|c|c|c|c|}
\hline \multicolumn{2}{|c|}{ INPUT } & \multicolumn{2}{c|}{ OUTFIT } & \multirow{2}{*}{ Measure } \\
\hline MNSQ & ZSTD & MNSQ & ZSTD & 0.00 \\
\hline 1.02 & 0.04 & 1.00 & -0.05 & 0.09 \\
\hline 0.06 & 0.45 & 0.06 & 0.38 & 0.30 \\
\hline 0.22 & 1.57 & 0.20 & 1.32 & 0.31 \\
\hline 0.23 & 1.63 & 0.21 & 1.38 & 0.42 \\
\hline 1.48 & 3.20 & 1.39 & 2.56 & -5.8 \\
\hline 0.71 & -2.63 & 0.71 & -2.47 & \\
\hline
\end{tabular}

Based on Table 10.1 and Table 10.2, it is known that the average value of MNSQ's INFIT and MNSQ's OUTFIT is close to 1 . Thus, the average student and item of this instrument fit the ideal criteria. Meanwhile, related to INFIT ZSTD and OUTFIT ZSTD, the average values of a person are -0.06 and -0.03 , respectively. While the value of INFIT ZSTD and OUTFIT ZSTD for each item is 0.05 and -0.05 .

Meanwhile, related to information on measurement results/measurement focus, the picture is obtained as shown in the following figure 3 .

Test Information Function

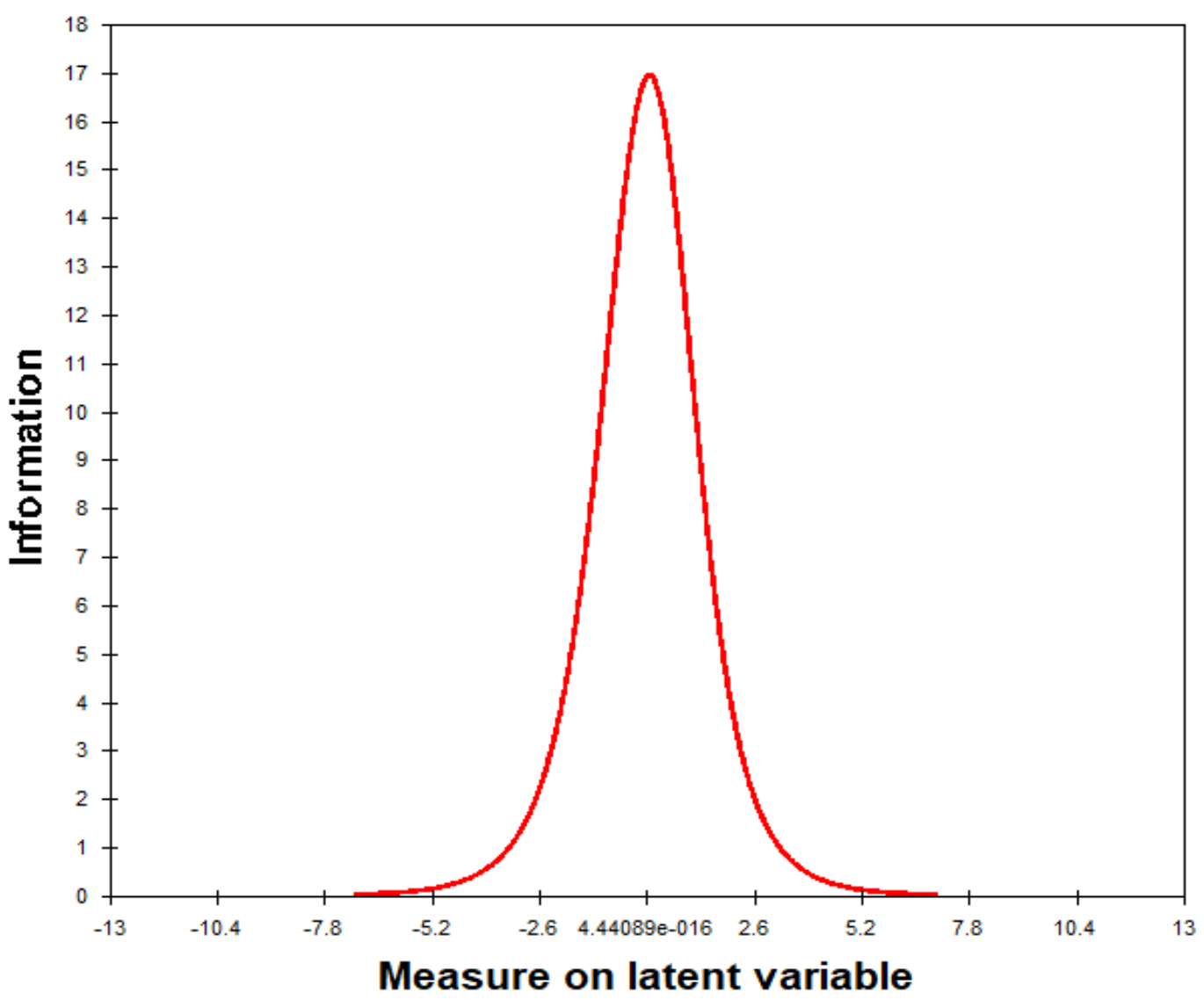

Figure 3. Information on measurement results 
The figure indicates that the item curiosity instrument questions students in elementary schools are more likely to produce high information on individuals with moderate abilities.

\section{Discussion}

According to Binson, curiosity is defined as the desire or need of someone to get answers to questions or things that cause it. Curiosity can produce an internal motivation to learn and understand something. Thus, it can be a fact that curiosity also affects the ability to understand children's concepts [4], [17]. The level of curiosity of a child can be identified through the spread of a research instrument with instrument data analysis through Rasch modeling because the measurement of content validity and instrument construct validity can be evaluated more precisely. Also, researchers can estimate the validity of respondents, namely by looking at respondents who have the most inconsistent answers [24]. Based on the results of the analysis of the instruments of elementary school students' curiosity about natural science subjects, overall these instrument items are easily agreed upon by students. Because of the average level of difficulty of standard the items are below the level of curiosity ability of elementary school students. This refers to the results of the analysis of the Output Table 3 data on the Wright Map Analysis (Person-Item Map) that map to determine the level of curiosity ability of students in elementary schools spread in a range of -1 to 3 logits. Their ability positions are mostly between -2 SD and + 2SD. The average logit of elementary school students' curiosity ability is +0.62 (check the output of Table 17 Measure Order in the appendix) is above the average logit item i.e. 0.00 . This means that the average logit curiosity ability of elementary school students is above the average level of difficulty of standard items. Meanwhile, the item difficulty level map is spread in the range -1 to 1 logit. Difficulty level position of 13 items (questions) is between-2SD - $+2 \mathrm{SD}$, from the data there are also no items outlier.

Meanwhile, the Cronbach Alpha Value, which represents the interaction between person and item items as a whole is included in a good category with a value of 0.74 , this means that the instruments and the students' abilities have complete integration. Furthermore, the value of Person Reliability as an indicator of the consistency of the respondent's answers of 0.70 , including enough categories. The average value of the person is smaller than the average item (where the average item is 0.00 logit) shows that the ability of participants, in general, is more small compared to the difficulty of the instrument items. While Item Reliability as an indicator of the quality of items in the instrument classified as a good category with a value of 0.88 . Other data in Table 10.1 and Table 10. 2 that can be known is the MNSQ INFIT and MNSQ OUTFIT both in the Person and Item tables. The criteria, the closer to number 1 the better, because the ideal value is 1 . From Table 4. Item Measure it is known that the average person is worth 1.00 and 1.00 while the average item is worth 1.02 and 1.00. Thus, the average person and infit/outfit items of MNSQ fit the ideal criteria. Meanwhile, related to INFIT ZSTD and OUTFIT ZSTD, the average values for a person are -0.06 and -0.03 , respectively. While the values for each item are 0.05 and -0.05 . The ideal value of ZSTD is 0 , the closer it is to 0 , the better. Thus, it can be said that the quality of the person and the items are good.

Finally, it is concerned with the separation or grouping of people and items. The separation index is a value to calculate strata of the level of difficulty of the questions that are useful for identifying groups of difficulty levels of the problem [24]. Individual separation shows how well a set of items in the elementary school curiosity enhancement instrument spreads across the range of logit skills. The greater the individual's separation, the better the instruments are arranged because the items in it can reach individuals with high to low-level abilities. Whereas the item separation shows how large the sample subject to measurement is spread along the linear interval scale. The higher the separation of items, the better the measurements are made. This index is also useful for defining the significance of the construct being measured.

From the output Table 10. Statistical Summary notes the separation for a person is 1.51 and for an item is 2.68 . The greater the separation value indicates the better instrument. A large separation value means the instrument can identify the group of respondents and item groups in more detail. Separation values are calculated more precisely through the formula: $\mathrm{H}=\{(4 \mathrm{x}$ separation $)+1\} / 3$ [24]. Thus, the separation value for a person is 2.35 rounded to 2 , while the separation for the items is 3.90 rounded to 4 . This implies that the study participants have a variety of abilities that can be categorized into four groups. Meanwhile, the level of difficulty items spread in 4 groups ranging from the easiest to the most difficult groups.

According to information on measurement results/measurement focus, it indicates that the item curiosity instrument questions students in elementary schools are more likely to produce high information on individuals with moderate levels of ability [24].

Furthermore, what is analyzed in Rasch modeling is unidimensionality which is seen as a tool to identify how consistent the indicators or dimensions are measured by the instrument. The results obtained turned out to be the value of Unexplained variance in the 1st to 5th contrast of residuals of more than $15 \%$, so that the dimensions were seen to be inconsistent in measuring instrument variables namely students' curiosity. The dimensions include epistemic curiosity, perceptual curiosity, specific curiosity, diverse curiosity. The results of the unidimensionality analysis have fulfilled the provisions so that the construct of the instrument used measures one variable, which is to find out the level of curiosity of students in elementary 
school grades 5 and 6 . This analysis uses the Output Table 2 by observing the value of Raw variance explained by measures and Unexplained variance in 1st to 5th contrast. Unidimensionality of measurement can be proven if Raw variance is explained by measures $\geq 20 \%$ (Note: general criteria for interpretation are: enough if $20-40 \%$, good if $40-60 \%$, and very good if above $60 \%$ ) and if Unexplained variance in 1 st to 5 th contrast of residuals $<15 \%$ each [24].

Meanwhile, for item analysis, the instrument items include the level of difficulty (item measure), the level of item suitability (item fit), and the detection of item bias.

First, the Item Difficulty Level Item can be reviewed in Table 4. Item Measure. It is known that 3 items are categorized as very difficult, namely items number 4.1 and 11. Hard categories have 4 items, namely numbers 10.7 .3 and 5. Easy categories are 4 items, namely 8,13,6 and 9 . Whereas there are 2 categories of very easy categories, namely numbers 12 and 2. From Table.4 it is known that the SD value is 0.30 This SD value if combined with the average logit value, the level of difficulty of items can be grouped into very difficult categories (greater $+1 \mathrm{SD})$, hard category $(0.0$ logit $+1 \mathrm{SD})$, easy category $(0.0$ logit $-1 \mathrm{SD})$, and very easy category (less than $-1 \mathrm{SD}$ ). Thus, the limit value for the very difficult category is more than 0.30 , the hard category is 0.00 to 0.30 , the easy category is -0.30 to less than 0.00 , and the very easy category is less than -030

Second, the level of suitability of the item. To see the suitability of the item with the model (item fit) which explains whether the item is functioning normally takes measurements so that there is no misconception in students of the item can be examined based on the data in Table 4 . Item Fit Order is the OUTFIT mean square (MNSQ) column, Z-standard OUTFIT (ZSTD), and point measure correlation (PT MEASURE CORR).

Criteria for checking item suitability or item incompatibility (outlier or misfit) according to Booner et al. (2014) are as follows: (1) MNSQ OUTFIT value is greater than 0.5 and smaller than 1.5 and the closer to 1 the better;

(2) ZFID OUTFIT value is greater than -2.0 and smaller than +2.0 , the closer to 0 the better; and (3) PT MEASURE CORR values are more than 0.4 and less than 0.85 . An item can be considered fit if it meets at least 1 of the 3 criteria [24].

Based on the 1st criteria, there are no misfit items. According to the 2nd standard, there are 3 misfit items, namely numbers 5,2 , and 1 . Whereas based on the $3 \mathrm{rd}$ criteria it is known that as many as 10 items have a PT MEASURE CORR value of more than 0.4 and less than 0.85 there are 3 items, namely numbered 2, 11 and 3 which have the value of PT MEASURE CORR in succession as follows: $0,36,0,36$ and 0,32 or less than 0,4 , so they are stated as an outfit. Referring to the views of Booner et al. (2014) then 13 items of statement items to find out the students' curiosity in elementary schools are declared fit in the sense of functioning normally and can be understood correctly by students in elementary schools and can measure what must be measured in this case is student curiosity.

Furthermore, a rating scale diagnosis is performed to find out whether participants understand the choice of answer choices on a scale of $1,2,3,4$, and 5 or not. The difference in answers is understood by respondents if the observed average and Andrich threshold values are in Table 6 which shows suitability and is equally increased in alternative answers 1, 2, 3, and 5 [24]. Whereas alternative answer 4 confuses respondents because the results of the analysis show that there is a lack of appropriateness in increasing the value of the two things in line with the increase in the rating scale. Thus, the difference in answer choices $1,2,3$, and 5 can be understood by participants, while answer choice 4 is less understandable. Thus, alternative answers are suggested not five but only four.

Next, to find out Primary School students' Curiosity based on Differential Item Functioning (DIF). One other measure of validity is that the instruments and items used do not contain bias, in the sense that it is more beneficial for one individual who has certain characteristics compared to individuals with other characteristics [22], [32]. An item statement is said to contain bias if the probability value of the item as stated in Output Table 7. Detection of Bias Items is below 0.05 [24]. In the context of this study, bias can only be seen from genders. The results of the analysis in Table 7 can be seen that 3 statement numbers have a gender bias, namely numbers 2 and 6 with probability values of 0.0007 and 0.0095 , respectively. Item number 2 and 6 are easier for female students to work on so that they benefit female students and tend to disadvantage male students. So for items 2 and 6, it is recommended to be deleted or replaced with other questions.

Another important thing in the Rasch Modeling is analyzing students' Abilities which are carried out on two things, namely the level of individual ability (person measure) and the level of individual suitability (person measure).

First, Analysis of Individual Ability. Data on the individual ability of elementary school students in grades 5 and 6 can be known from Table 8. Student Ability. From this table, the $\mathrm{SD}$ value is 0.56 and the mean logit (mean) is 0.62 . Thus, the individual abilities of elementary school students in grades 5 and 6 can be grouped by looking at the logit score limit into the high ability category (greater than $0.62+0.56=1.18$ ), the medium ability category (between $0.62-0,56$ and $0.62+0.56$ or 0.06 and 1.18), and the category of low ability (less than $0.62-0.56=0.06$ ). By looking at the logit value of each grade 5 and 6 elementary school students in Table 8 in the Measure column, it can be seen from the total number of participants as many as 99 people, there are 14 people included in the high ability category, 69 people in the medium ability category and 16 people in the low ability category. In Table 8 shows that the level of curiosity of students in grades 5 and 6 at SDN 
Dadaha and MIN 1 in Tasikmalaya in science subjects is mostly at a moderate level (69.69\%), meaning students in grades 5 and 6 in SDN Dadaha and MIN 1 in Tasikmalaya: a) tends to hesitate to understand the views of others in an instrument condition (curiosity) so that students have not shown optimal confidence, b) students show their openness with others but are still shy c) some students have shown sensitivity to the needs/desires of others, d) some students already trust each other and understand and work together / collaborate well [33]. Second, the level of suitability of an individual to see the suitability of an individual's response based on his ability to the ideal model (person fit) can be analyzed based on the mean squire (MNSQ) OUTFIT, Z-standard OUTFIT (ZSTD), and point measure correlation (PT MEASURE CORR). A participant can be considered fit if it meets at least 1 of the 3 criteria [24].

Based on these criteria it is known that as many as 94 elementary school students in grades 5 and 6 are declared fit in the sense of giving answers according to their level of ability. While the other 5 students gave answers that were less appropriate for their level of ability, namely numbers: $12,27,65,89$, and 48 . They were the possibility of guessing an answer or not being sincere in answering. To overcome this problem is to create opportunities for individuals to indulge in intense learning about their specific disciplinary topics, increased curiosity and the development of long-term interests in science can occur. [8].

Overall from the results of the analysis of curiosity of elementary school students in Class 5 and 6 , it can be concluded that the overall instrument is proven to have a good construction so that it can be used for instruments in the assessment and research.

\section{Conclusions}

Based on the results of the analysis of the instruments processed through the Rasch model it can be concluded that this instrument can measure the curiosity of elementary school students towards natural science subjects so that it can be used as a benchmark tool for study program managers to assess the level of success of the science learning process in elementary school. The analysis shows that the value of reliability is acceptable (Alpha Cronbach), including the good category. Although 2 items do not meet the standard criteria as a measurement tool, namely number 2 and number 6 bias genders because it is more beneficial for women. Furthermore, the value of Person Reliability as an indicator of the consistency of the answers of respondents, including enough categories. This was considered enough because as many as 94 elementary school students grade 5 and 6 were declared fit, in the sense of being able to provide answers according to their level of ability. While 5 other students answered less following the level of ability, the possibility of the five students guessing the answer or not giving answers. While Item Reliability as an indicator of the quality of items in the instrument is classified as a good category. Because items of an instrument of curiosity of students in elementary schools are more likely to produce high information on individuals with moderate levels of ability with all 13 items of statement declared fit and the average level of difficulty of standard the items are below the level of ability of students, this means that the instrument is functioning normally and can be approved is also understood correctly by students in elementary schools.

\section{Recommendation}

To increase students' curiosity in natural science lessons, there needs to be aware of and good relationships between teachers and students. The use of instruments can assist teachers in investigating students' level of curiosity, making it easier for teachers to make improvements to the learning process of natural science.

\section{Acknowledgments}

Thank you to the Lecturer in Learning Evaluation in Elementary School, Universitas Pendidikan Indonesia for allowing me to make this article.

\section{REFERENCES}

[1] S. A. Kamaruddin, "Character Education and Students Social Behavior,” J. Educ. Learn., vol. 6, no. 4, pp. 223-230, 2012.

[2] A. Adams, "The Need For Character Education," Int. J. Soc. Sci. Humanit. Stud., vol. 3, no. 2, pp. 23-32, 2011.

[3] T. M. Amabile, K. G. Hill, B. A. Hennessey, and E. M. Tighe, "The Work Preference Inventory: Assessing Intrinsic and Extrinsic Motivational Orientations," J. Pers. Soc. Psychol., vol. 66, no. 5, pp. 950-967, 1994.

[4] B. Binson, "Curiosity-based learning (CBL) program," US-China Educ. Riview, vol. 6, no. 12, pp. 13-22, 2009.

[5] S. Kedge and B. Appleby, "Promoting curiosity through the enchancement of competetence," Br. J. Nurs., vol. 19, no. 9, pp. 584-587, 2010.

[6] P. Marilyn, B. L. Grabowski, and C. P. Rynd, "Curiosity as a Personality Variable Influencing Learning in a Learner Controlled Lesson With and Without Advisement," ETR\&D, vol. 42, pp. 5-20, 1994.

[7] H. L. Pluck, G \& Johnson, "Stimulating curiosity to enhance learning," GESJ Educ. Sci. Psychol., vol. 2 (19), no. 15121801, 2011.

[8] T. Post and J. H. W. Van Der Molen, "Development and 
validation of a questionnaire to measure primary school children' $\mathrm{s}$ images of and attitudes towards curiosity ( the CIAC questionnaire )," J. Motiv. Emot., vol. 43, no. 1, pp. 159-178, 2019.

[9] J. L. Weible and H. T. Zimmerman, "Science curiosity in learning environments: developing an attitudinal scale for research in schools , homes , museums , and the community," Int. J. Sci. Educ., vol. 0693, no. June, 2016.

[10] M. W. Gallagher, S. J. Lopez, M. W. Gallagher, and S. J. Lopez, "Curiosity and well-being," J. Posit. Psychol., vol. 2 no 4, pp. 236-248, 2007.

[11] T. B. Kashdan, P. Rose, and F. D. Fincham, "Curiosity and Exploration: Facilitating Positive Subjective Experiences and Personal Growth Opportunities Curiosity and Exploration: Facilitating Positive Subjective Experiences and Personal Growth Opportunities," J. Personal. Assesment, no. March 2015, pp. 37-41, 2010.

[12] R. P. Collins, J. A. Litman, and C. D. Spielberger, "The measurement of perceptual curiosity," Pers. Individ. Differ., vol. 36, pp. 1127-1141, 2004.

[13] G. Loewenstein, "The Psychology of Curiosity: A Review and Reinterpretation," Psychol. Bull., no. July 1994, 2014.

[14] S. Von Stumm and S. Broome, The power of curiosity, no. June. 2012

[15] M. K. Noordewier and E. Van Dijk, "Curiosity and time : from not knowing to almost knowing," J. Cogn. Emot., vol. 9931, no. March 2016.

[16] D. E. Berlyn, "A theory of human curiosity," Br. J. Psychol., vol. 3, 1954.

[17] A. Y. Arditama, S. Wardani, E. Purwanti, and N. Hindarto, "Storybook Influence on Science Concept Comprehension Through Curiosity of Fifth Grade Elementary School Student," J. Prim. Educ., vol. 7, no. 1, pp. 1-9, 2018.

[18] M. Lindholm, "Promoting Curiosity? Possibilities and Pitfalls in Science Education," Sci. Educ., no. 1, pp. 987$1002,2018$.

[19] U. H. Latifah and D. B. Widjajanti, "Pengembangan Bahan Ajar Statistika dan Peluang Berbasis Multiple Intelligences Berorientasi pada Prestasi , Pemecahan Masalah , dan Rasa Ingin Tahu," J. Ris. Mat. 4, vol. 4, no. 2, pp. 176-185, 2017.

[20] B. Gurning and A. Siregar, "The Effect of Teaching Strategies and Curiosity on Students Achievement in Reading Comprehension," English Lang. Teach., vol. 10, no. 11, pp. 191-198, 2017.

[21] T. B. Kashdan, M. W. Gallagher, P. J. Silvia, and M. Steger,
"The Curiosity and Exploration Inventory-II: Development, Factor Structure, and Psychometrics," J. Res. Pers., no. December, p. 12, 2009.

[22] R. L. Lamb, L. Annetta, and J. Meldrum, "Measuring science interest: Rasch validation of the science interest survey," no. June, 2011.

[23] O. Spektor-levy and Y. K. Baruch, "Science and Scientific Curiosity in Pre-school - The teacher' s point of view Ornit Spektor-Levy , Yael Kesner Baruch \& Zemira Mevarech (2011): To link to this article : http://dx.doi.org/10.1080/09500693.2011.631608 Pre-print version Science and Scien," Int. J. Sci. Educ., vol. 35(13), no. January, 2011.

[24] B. Sumintono and W. Widhiarso, Aplikasi Pemodelan Rasch Pada Assesment Pendidikan. 2015.

[25] L. B. Sousa, G. Prieto, and M. Vilar, "The Adults and Older Adults Functional Assessment Inventory: A Rasch Model Analysis," Res. Anging, pp. 1-28, 2014.

[26] H. E. Goh, I. Marais, and M. J. Ireland, "A Rasch Model Analysis of the Mindful Attention Awareness Scale," Assesment, pp. 1-12, 2015.

[27] R. S. Timofte and L. Siminiciuc, "Utilisation Of R Asch Model For The Analysis Of An Instrument Developed By Mapping Items To Cognitive Levels Of Marzano Taxonomy," Acta Didact. Napocensia, vol. 11, no. 2, pp. 71-78, 2018

[28] C. J. Camp, J. R. Rodrigue, and K. R. Olson, "Curiosity in Young, Midle-Aged, and Older Adult," Edicational Gerontol., vol. 10 no 5, pp. 37-41, 2015.

[29] K. A. C. Creswell, "Creswell's Appreciation Of Arabian Architecture,” JSTOR, vol. 8, no. 1991, pp. 94-102, 2016.

[30] W. A. Collins, Development During Middle Childhood: The Years From Six to Twelve. 1984.

[31] G.-C. I. S. District, "Developmental Characteristics of Fifth Graders The Ten -Year-Old The Eleven -Year-Old," Curriculum Guides and Developmental Characteristics. pp. $1-2,2002$.

[32] A. Taufik, E. S. Yudha, and D. Suryana, "The Raven' s Advanced Progressive Matrices in Education Assessment with a Rasch Analysis," Univers. J. Educ. Res., vol. 7, no. 9, pp. 1996-2002, 2019.

[33] U. Suherman, N. Budiman, D. Suryana, E. S. Yudha, A. B. Ahmad, and N. Bin Saper, "Dimension of Peace Culture Based on Al-Quran Values," Univers. J. Educ. Res., vol. 7, no. 10, pp. 2171-2178, 2019. 